OPEN

SUBJECT AREAS:

VIROLOGY

PATHOGENS

PATHOLOGY

EXPERIMENTAL ORGANISMS

Received

25 April 2012

Accepted

28 September 2012

Published

15 November 2012

Correspondence and requests for materials should be addressed to

H.M.W. (hana. weingart|@inspection. gc.ca) or G.K. (gary. kobinger@phac-aspc.

gc.ca)

\section{Transmission of Ebola virus from pigs to non-human primates}

\author{
Hana M. Weingartl',2, Carissa Embury-Hyatt ${ }^{1}$, Charles Nfon ${ }^{1}$, Anders Leung ${ }^{3}$, Greg Smith' \\ \& Gary Kobinger ${ }^{3,2}$
}

\begin{abstract}
${ }^{1}$ National Centre for Foreign Animal Disease, Canadian Food Inspection Agency, 1015 Arlington St. Winnipeg, Manitoba, R3E $3 \mathrm{M4}$, Canada, ${ }^{2}$ Department of Medical Microbiology, University of Manitoba, Winnipeg, Canada, ${ }^{3}$ National Microbiology Laboratory, Public Health Agency of Canada, 1015 Arlington St., Winnipeg, Manitoba, R3E 3R2, Canada.
\end{abstract}

Ebola viruses (EBOV) cause often fatal hemorrhagic fever in several species of simian primates including human. While fruit bats are considered natural reservoir, involvement of other species in EBOV transmission is unclear. In 2009, Reston-EBOV was the first EBOV detected in swine with indicated transmission to humans. In-contact transmission of Zaire-EBOV (ZEBOV) between pigs was demonstrated experimentally. Here we show ZEBOV transmission from pigs to cynomolgus macaques without direct contact. Interestingly, transmission between macaques in similar housing conditions was never observed. Piglets inoculated oro-nasally with ZEBOV were transferred to the room housing macaques in an open inaccessible cage system. All macaques became infected. Infectious virus was detected in oro-nasal swabs of piglets, and in blood, swabs, and tissues of macaques. This is the first report of experimental interspecies virus transmission, with the macaques also used as a human surrogate. Our finding may influence prevention and control measures during EBOV outbreaks.

bola viruses belong to the family Filoviridae, genus Ebolavirus. Those endemic to Africa cause severe hemorrhagic fever with frequent fatal outcome in humans, great apes and several species of non-human primates (NHPs). Fruit bats are considered to be the natural reservoir for EBOV in Africa ${ }^{1}$. In 2009, the only non-African known species of EBOV, Reston Ebola virus (REBOV), was isolated from swine in Philippines, with antibodies against the virus detected in pig farmers ${ }^{2,3}$. However REBOV did not cause clinical signs in experimentally inoculated pigs $^{4}$. In contrast to African species of EBOV, REBOV does not cause clinical symptoms in humans, although the infection may be fatal in cynomolgus macaques ${ }^{5}$. We have previously demonstrated that Zaire-EBOV (ZEBOV) can infect pigs, cause disease, and transmit to in-contact pigs ${ }^{6}$. While primates develop systemic infection associated with immune dysregulation resulting in severe hemorrhagic fever, the EBOV infection in swine affects mainly respiratory tract, implicating a potential for airborne transmission of $\mathrm{ZEBOV}^{2,6}$. Contact exposure is considered to be the most important route of infection with EBOV in primates ${ }^{7}$, although there are reports suggesting or suspecting aerosol transmission of EBOV from NHP to NHP ${ }^{8-10}$, or in humans based on epidemiological observations ${ }^{11}$. The present study was design to evaluate EBOV transmission from experimentally infected piglets to NHPs without direct contact.

\section{Results}

Six four-week old Landrace piglets (Sus scrofa) were oronasally inoculated with $10^{6} \mathrm{TCID}_{50}$ of ZEBOV (Kikwit 95) per animal. The piglets were transferred to a separate room for the inoculations, and then moved back into the room containing four cynomolgus macaques. This age group was selected based on the previous observation of differences in severity of the disease in ZEBOV inoculated piglets ${ }^{6}$ to ensure sufficient survival time of the piglets potentially needed for virus transmission, and to determine whether piglets without an overt clinical disease could transmit the virus. The macaques were housed in two levels of individual cages inside the pig pen, and separated from the piglets by wire barrier placed about $20 \mathrm{~cm}$ in front of the bottom cages to prevent direct contact between the two species. Bottom cages housing NHPs Nos. $07 \mathrm{M}$ and $20 \mathrm{~F}$ were about $10 \mathrm{~cm}$ above the ground, top cages housing NHPs Nos. 34F and 51M were about $1.4 \mathrm{~m}$ above the ground. The NHP cages were located immediately to the side of the air exhaust system. The cubicle layout respective to the airflow (ten complete air exchanges per hour) in the room is schematically indicated in Supplemental Figure S1. During the husbandry, piglets were moved away from the cages and enclosed by the gate system. The floor was washed, taking care that the water is sprayed at low pressure and away from the NHP cages, to avoid any splashes into the bottom cages. Also the 


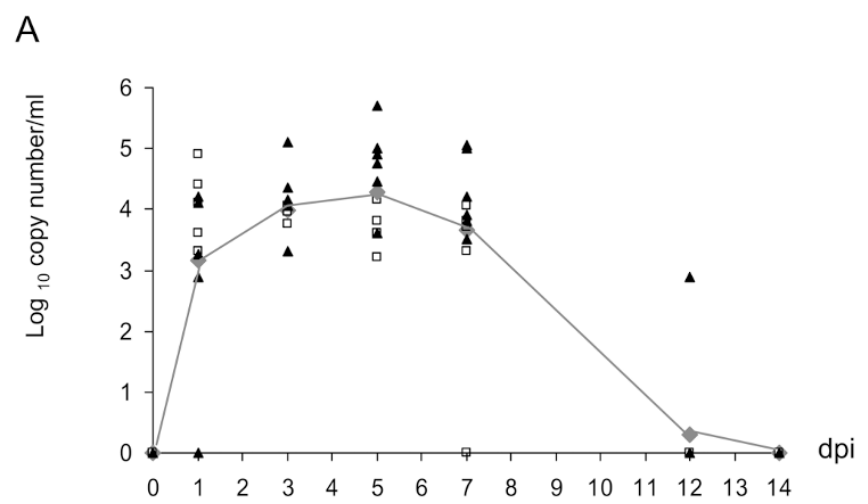

B

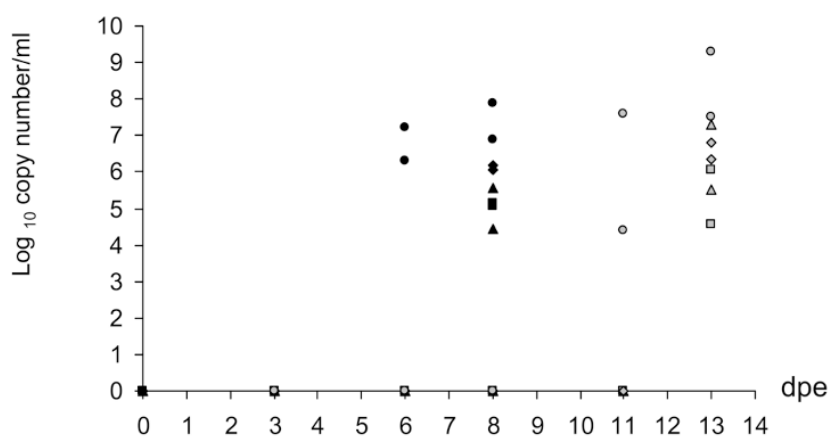

Figure $1 \mid$ Detection of EBOV RNA in swabs and blood. (A) Shedding in pigs. Squares represent the oral swabs and triangles illustrate the nasal swabs. Gray line with diamonds shows the general trend of the oro-nasal shedding. (B) Non-human primates: square markers represent the oral swabs, diamonds represent the rectal swabs, triangles represent the nasal swabs, circles represent blood samples. Gray markers-NHP No. 51M and 20F, black markers-NHP 07M and 34F. "dpi” (days post inoculation) and "dpe" (days post exposure) on the $\mathrm{X}$ axis are equivalent.

$20 \mathrm{~cm}$ space between the wire barrier and the cages was cleaned separately with running water prior to proceeding with NHP cage cleaning. Both animal species were fed after the cleaning, providing new clean dishes for the macaques, with staff changing disposable outer gloves between procedures and animals. The design and size of the animal cubicle did not allow to distinguish whether the transmission was by aerosol, small or large droplets in the air, or droplets created during floor cleaning which landed inside the NHP cages (fomites). The husbandry flow during the sampling days was: cleaning, followed by sampling, then feeding, with staff changing disposable outer gloves between procedures and animals. Pigs and NHPs were sampled on alternative days except for day 3 post infection, when NHPs were sampled in the morning and the piglets in the afternoon.

Clinical signs and gross pathology in swine, following the inoculation with EBOV, were comparable to previous infection study in piglets of this age group ${ }^{6}$. Increase in respiratory rate (up to 80 breaths $/ \mathrm{min}$ ) and in rectal temperatures $\left(40.2-40.5^{\circ} \mathrm{C}\right.$ ) was observed between 5 and 7 days post infection (dpi). All piglets apparently recovered from the disease by 9 dpi. Piglets Nos. 1, 2 and 4 were euthanized at $12 \mathrm{dpi}$, and piglets Nos. 3, 5 and 6 at $14 \mathrm{dpi}$, based on experimental schedule. Clinical scores and parameters are provided in the Supplementary Information (Supplemental Figure 2A, Supplemental Table 1). No significant lesions were observed at the necropsy. Microscopic lung lesions were focal and not extensive,

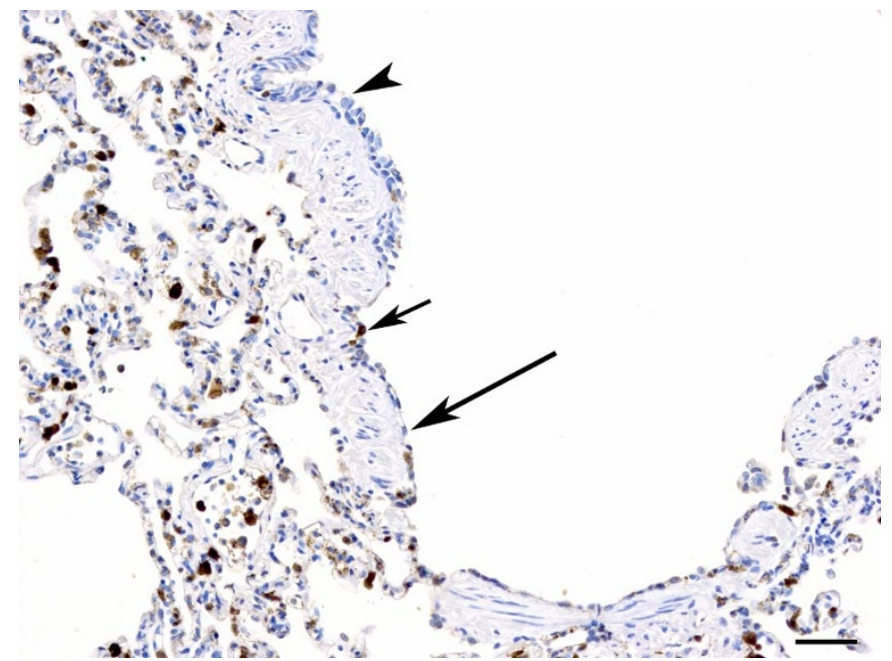

Figure $2 \mid$ Lungs, macaque No.34F. Segmental attenuation and loss of respiratory epithelium in the bronchiolar wall (large arrow) with some areas of the lungs relatively unaffected (arrowhead). Immunostaining for Ebola virus antigen was detected in occasional respiratory epithelial cells (small arrow) as well as within alveolar and septal macrophages.

Bar $=50 \mu \mathrm{m}$.

characterized by broncho-interstitial pneumonia with a lobular pattern, similar to those described in our previous report ${ }^{6}$. Virus antigen was detected by immunohistochemistry in three piglets (No. 2, 4, and week signal in No. 5), primarily within the areas of necrosis often adjacent to bronchioles (Supplemental Figure S3A). The presence of virus in the lung was confirmed by detection of EBOV RNA employing real-time RT-PCR targeting the $\mathrm{L}$ gene, and by virus isolation on Vero E6 cells for piglet No. 2 and No. 4. Virus isolation was also attempted from lung associated lymph nodes, based on detection of viral RNA, yielding one, successful isolation. Viral RNA was detected in submandibular lymph nodes of all piglets, and in the spleen and liver of two piglets. Low level of viremia based on RNA levels was detected in blood of four piglets at 5 and 7 dpi. EBOV RNA was detected in nasal and oral swabs of piglets from 1 dpi until $7 \mathrm{dpi}$, inclusively (Figure 1A), and from rectal swabs on day 1 and 5, but not at 3, 7 and 12 dpi (Supplemental Table 1). Viral isolation was attempted on all swabs. Out of 45 oral and nasal swabs positive by RT-PCR, 16 were positive on virus isolation, while two out of 11 RNA-positive rectal swabs tested positive for virus. Presence of EBOV RNA in cell culture supernatants from the isolates with observed CPE was confirmed by real time RT-PCR (Supplemental Table 1; Supplemental Table 2).

Air sampling was conducted on day $0,3,6,8$ and 11 post inoculation. Real time RT-PCR targeting the L gene detected viral RNA on days 6 and 8 post inoculation. Location in front of the bottom cages at about $75 \mathrm{~cm}$ above the floor was sampled in $30 \mathrm{~min}$ triplicates following husbandry, during the NHP sampling. Average values of 4.4 $\log _{10}$ copies/ml and $3.85 \log _{10}$ copies/ml of the sampling buffer were detected at 6 and $8 \mathrm{dpi}$, respectively. Virus isolations were not successful, likely due to the sampling buffer composition $(0.1 \%$ Tween 20).

All four NHPs (Macaca fascularis) were alert and in good apparent health until 7 days post exposure (dpe - corresponding to dpi of piglets) with ZEBOV. At 8 dpe, macaques 07M (bottom left cage) and $34 \mathrm{~F}$ (upper right cage), housed in cages located within an air flow towards the exhaust system, were euthanized based on clinical signs typical for EBOV infection in NHPs. Both had petechial hemorrhages on the skin of the chest and along internal surfaces of the arms and legs. Macaques 51M and 20F were visually healthy until 12 dpe, when early clinical signs were noted, and both animals were 
euthanized the next day ( 13 dpe). The NHPs were euthanized when convincing clinical signs typical for EBOV infection became apparent, preferably prior to the humane endpoint (Supplemental Figure S2B; Supplemental Table 1). Examination of internal organs at the necropsy exposed damages mainly to the lung (Supplemental Figure S4) and liver. Microscopic lesions and antigen distribution in the organs were similar to previous reports ${ }^{12-14}$, except for the lesions and antigen distribution in lungs. Interstitial pneumonia was characterized by thickened and hypercellular alveolar septa due to infiltration by primarily macrophages (Supplemental Fig. 3B), with multifocal areas of alveolar hemorrhage and edema. EBOV antigen was detected extensively in alveolar and septal macrophages using double immunostaining (Supplemental Fig. 3C), as well as within pneumocytes and endothelial cells. Viral antigen was also observed within bronchiolar epithelial cells with adjacent segmental loss of epithelial cells (Figure 2.) and within respiratory epithelial cells of the trachea. The pattern of lesions and immunostaining for EBOV antigen in lungs suggests infection of the lungs both, via respiratory epithelium and due to viremic spread of the virus.

There was a remarkable difference in the type and quantity of cells infiltrating the lungs between the macaques and the pigs, although viral antigen was detected only in alveolar macrophages of both species. Monocytes/macrophages were essentially the only leukocyte type infiltrating the lungs in non-human primates, while large quantities of non-infected lymphocytes were recruited into the pig lungs. This phenomenon can be linked to different clinical picture in the two animal species: respiratory distress in pigs (severe in a specific age group ${ }^{6}$ ) versus systemic disease with no major respiratory signs in NHPs. It will be important to identify differences and similarities in ZEBOV-induced pathogenesis and pathology between the two species in future studies.

Infection of the NHPs with ZEBOV was confirmed by detection of viral RNA (real time RT-PCR targeting the L gene), and in all samples collected at euthanasia by virus isolation. The first detection of ZEBOV RNA was in the blood of NHPs $34 \mathrm{~F}$ and $07 \mathrm{M}$ at 6 dpe, with virus isolation from macaque 07M. This was followed by ZEBOV RNA detection in nasal, oral and rectal swabs from the same NHPs at 8 dpe (Figure 1B). A similar pattern was observed for macaques $51 \mathrm{M}$ and $20 \mathrm{~F}$, starting at 11 dpe with detection of RNA in blood and virus isolation from animal $20 \mathrm{~F}$, followed by RNA and virus detection in swabs at 13 dpi. Detection of viral RNA and infectious virus in blood, swabs and tissues of the macaques (summarized in Supplemental Table 4) confirmed systemic spread of the virus. Whole genome sequencing performed on virus nucleic acid from selected swab and lung samples from pigs and NHPs confirmed identity of the virus.

\section{Discussion}

Pigs were the source of ZEBOV at a time of infection of NHPs euthanized at 8 dpe $(07 \mathrm{M}$ and $34 \mathrm{~F})$ since shedding from the macaques was not detected at dpe 3 or 6 . NHPs euthanized at 13 dpe (20F, 51M) could have contracted ZEBOV from the environment contaminated by either species, considering previous reports on development of disease following aerosol exposure ${ }^{10}$, or other inoculation routes ${ }^{5,15,16}$, although pigs can generate infectious short range large aerosol droplets more efficiently then other species ${ }^{17}$. We have also never observed transmission of EBOV from infected to naive macaques, including in an experiment employing the same cage setting as in the current study, where three NHPs intramuscularly inoculated with EBOV did not transmit the virus to one naive NHP for 28 days, the duration of the protocol. During another study, three EBOV infected NHPs cohabiting with 10 naive NHPs in adjacent cage systems did not transmit the virus to naive animals for 28 days (unpublished data). The exact route of infection of the NHPs is impossible to discern with certitude because they were euthanized at a time when EBOV had already spread systemically. However, the segmental attenuation and loss of bronchiolar epithelium and the presence of Ebola virus antigen in some of the respiratory epithelial cells in the lungs of all macaques suggest that the airways were one of the routes involved in the acquisition of infection, consistent with previous reports ${ }^{9,10}$. Other routes of inoculation generally did not lead to lesions in the respiratory tract comparable to those observed in this study ${ }^{12,13}$.

Under conditions of the current study, transmission of ZEBOV could have occurred either by inhalation (of aerosol or larger droplets), and/or droplet inoculation of eyes and mucosal surfaces and/or by fomites due to droplets generated during the cleaning of the room. Infection of all four macaques in an environment, preventing direct contact between the two species and between the macaques themselves, supports the concept of airborne transmission.

It is of interest, that the first macaques to become infected were housed in cages located directly within the main airflow to the air exhaust system. The experimental setting of the present study could not quantify the relative contribution of aerosol, small and large droplets in the air, and droplets landing inside the NHP cages (fomites) to EBOV transmission between pigs and macaques. These parameters will need to be investigated using an experimental approach specifically designed to address this question.

The present study provides evidence that infected pigs can efficiently transmit ZEBOV to NHPs in conditions resembling farm setting. Our findings support the hypothesis that airborne transmission may contribute to ZEBOV spread, specifically from pigs to primates, and may need to be considered in assessing transmission from animals to humans in general. The present experimental findings would explain REBOV seropositivity of pig farmers in Philippines ${ }^{2,3}$ that were not involved in slaughtering or had no known contact with contaminated pig tissues. The results of this study also raise a possibility that wild or domestic pigs may be a natural (nonreservoir) host for EBOV participating in the EBOV transmission to other species in sub-Saharan Africa.

\section{Methods}

Virus. ZEBOV strain Kikwit 95 was produced on VERO E6 cells in minimal essential medium (MEM) supplemented with $2 \%$ fetal bovine serum and antibiotics (Penicillin/Streptomycin). Virus titers were determined by standard TCID S0 and/or immunoplaque assays on VERO E6 cells. Procedures for the production and propagation of ZEBOV and all subsequent experiments involving infectious materials were performed in the Containment Level (CL) 4 facilities of the Canadian Science Center for Human and Animal Health (CSCHAH).

Animal experiments. Four cynomolgus macaques were acclimatized in the BSL4 animal facility for two weeks, and housed in the same room for one week prior to the swine inoculation. The macaques were housed in two levels of individual cages inside the pig pen, and separated from the piglets by wire barrier placed about $15 \mathrm{~cm}$ in front of the cages to prevent direct contact between the two species. Bottom cages housing NHPs Nos. $07 \mathrm{M}$ and $20 \mathrm{~F}$ were about $20 \mathrm{~cm}$ above the ground, while top cages housing NHPs Nos. 34F and 51M were about $1.4 \mathrm{~m}$ above the ground. The NHP were sampled at 3 and $6 \mathrm{dpi}$ (nasal, oral rectal swabs, blood) as per experimental schedule. Two macaques were euthanized for humane reasons at 8 days post exposure (dpe), and all animals were sampled at that time. Two remaining NHPs were in addition sampled at $11 \mathrm{dpe}$, and at 13 dpe when they were euthanized. The animals were euthanized when typical clinical signs of Ebola infection became apparent, if possible prior to reaching the humane endpoint. Lung, lung associated lymph nodes, liver, spleen and intestine were collected at the necropsy.

Pigs (breed Landrace) were obtained from a high health status herd operated by a recognized commercial supplier in Manitoba, Canada. Three-week old piglets, designated as animal No. 1-6, were acclimatized for seven days prior to the inoculation in an animal cubicle already housing the non-human primates. The six piglets were inoculated oro-nasally with $2 \mathrm{ml}$ of $10^{6} \mathrm{TCID}_{50}$ total per animal $(0.5 \mathrm{ml}$ per each nostril and $1 \mathrm{ml}$ orally) in a room adjacent to the BSL4 animal cubicle and subsequently housed in proximity to cages with four non-human primates (NHP). Swine rectal temperatures were taken during the sampling performed under anesthesia on days $0,1,3,5,7,12$ and 14 , when blood and rectal, oral and nasal swabs were collected. Three piglets were euthanized on day 12 post inoculation (no. $1 \mathrm{M}, 2 \mathrm{M} .4 \mathrm{~F}$ ), and three on day $14(3 \mathrm{M}, 5 \mathrm{~F}, 6 \mathrm{~F})$, as per experimental schedule. Muscle, lung, liver, spleen, trachea, and submandibular, lung associated and mesenteric lymph nodes were collected at necropsy.

All animal manipulations were performed under CL4 conditions and followed Animal Use Document No. CSCHAH AUD\# C-11-004 approved by the Animal Care 
Committee of the Canadian Science Centre for Human and Animal Health, according to and following the guidelines of the Canadian Council on Animal Care.

Virus isolation. Swabs collected into $1 \mathrm{ml}$ of cMEM, blood, and tissues homogenized in MEM using a bead mill homogenizer according to the manufacturer's protocol (Tissue Lyser, Qiagen) were used for virus isolation and real time RT-PCR analysis. All NHP samples and swine rectal swabs were plated in 10-fold serial dilutions of supernatant on Vero E6 cells with six replicates per dilution. At 72-96 h postinfection the plates were scored for cytopathic effect $(\mathrm{CPE})$ and $\mathrm{TCID}_{50}$ virus titers were calculated using the Reed and Muench method. Swine rectal swabs had to be however carried over onto replica plates for three passages prior to reading the CPE. Swine nasal and oral swabs, blood and tissues were first analyzed by real time RT-PCR targeting the ZEBOV L gene, followed by virus isolation on Vero E6 cells in P6 plates on selected samples.

Virus RNA detection. NHP samples: Total RNA was isolated from tissues preserved and homogenized in RNA later employing the RNeasy Mini Kit (QIAGEN). RNA from nasal washes and swabs was isolated using the QIAamp Viral RNA Mini Kit (QIAGEN, GmbH).

Swine samples: RNA was isolated using Tripure Reagent (Roche Applied Science) according to the manufacturer's recommendations from swabs, blood or $10 \% \mathrm{w} / \mathrm{v}$ tissue homogenates in cMEM. One-Step real-time RT-PCR was carried out using following primers and probe:

ZebovForward -CAGCCAGCAATTTCTTCCAT;

ZebovReverse- TTTCGGTTGCTGTTTCTGTG;

ZebovProbe FAM-ATCATTGGCGTACTGGAGGAGCAG-NFQ.

Armoured enterovirus RNA (Asuragen) was used as external extraction/reaction control. Quantitect Reverse Transcriptase Real-time PCR kit (Qiagen) was employed for the PCR reactions according to the manufacturer's specifications. Reaction conditions for the RT-PCR were as follows: $50^{\circ} \mathrm{C}$ for 30 minutes; $95^{\circ} \mathrm{C}$ for 15 minutes; 45 cycles of $95^{\circ} \mathrm{C}$ for 15 seconds followed by $60^{\circ} \mathrm{C}$ for 45 seconds. The samples were run on the Rotor-Gene 6000 (Qiagen) or on the the LightCycler 480 (Roche Applied Science). Copy numbers were determined based on the L-gene Ebola plasmid standard control curve. Cut off value for samples to be considered positive were 3 $\log _{10}$ copies/ml (Rotorgene) or $3.15 \log _{10}$ copies/ml (LightCycler 480 ).

Air sampling. The air was sampled using BioCapture 650 Air Sampler (FLIR, Arlington, VA) on days $0,3,6,8$ and 11 post inoculation of the piglets. The air sampling started after husbandry, concurrent to NHP sampling, later in the morning before noon. Location in front of the bottom cages at about $75 \mathrm{~cm}$ above the floor was sampled in 30 min triplicates. The collection took place over a span of about two hours in total (three 30 min collection times with changes of cartridges in between). The air sampler device collects particles by bubbling the air through a pre-loaded buffer $(0.74 \%$ Tris/ 0.1 Tween 20$)$ provided in a sealed cartridge by the manufacturer. This solution is not optimal for recovery of live enveloped viruses, and virus isolation attempts were unsuccessful. ZEBOV RNA was detected by real time RT-PCR targeting the $\mathrm{L}$ gene.

EBOV sequencing. Viral RNA previously extracted for real time PCR was sequenced by first generating cDNA with the use of Omniscript reverse transcriptase (Qiagen) and random hexamers along with specific EBOV primers followed by PCR with iProof high fidelity DNA polymerase (Bio-Rad) with specific primers (available upon request). DNA sequencing was carried out using the $3730 x \mathrm{DNA}$ Analyzer (ABI).

Histology and immunohistochemistry. Tissues were fixed in $10 \%$ neutral phosphate buffered formalin, paraffin embedded using standard procedures, sectioned at $5 \mathrm{~m}$, and stained with hematoxylin and eosin (HE) for histopathologic examination. Detection of viral antigen was performed using A 1:2000 dilution of rabbit polyclonal anti-ZEBOV VP40 antibody as described previously ${ }^{6}$. Identification of macrophages in the lungs was performed by immunostaining for the macrophage/monocyte marker L1 using Clone Mac387 (Dako, USA) primary antibodies. The tissue sections were quenched for 10 minutes in aqueous $3 \%$ hydrogen peroxide, prior to retrieval of epitopes using high pH AR10 (BioGenex, CA) in a BioCare Medical Decloaking Chamber. Antibody Clone Mac 387 was applied for 10 minutes at a dilution of 1:3200, and visualized using an AP-polymer kit, Mach 4 Universal (BioCare Medical, $\mathrm{CA})$ for 30 minutes, and reacted with Vulcan Fast Red (BioCare Medical, CA) substrate. For the Mac387/Ebola double stain, antibody Clone Mac 387 was applied for 10 minutes at a dilution of 1:3200, and visualized using a multilink horseradish peroxidase labeled kit, Super Sensitive Link-Label IHC Detection System (BioGenex, $\mathrm{CA})$, reacted with the chromogen diaminobenzidine (DAB). The sections were then incubated with a denaturing solution (1 part A, 3 parts B, BioCare Medical, CA) for 5 minutes, pretreated with proteinase $\mathrm{K}$ enzyme for 10 minutes, and rabit polyclonal anti-Ebola Zaire VP40 antibody was applied to the sections at a 1:2,000 dilution for one hour. The anti-EBOV antibody was visualized using an AP-polymer kit, Mach 4
Universal (BioCare Medical, CA) for 30 minutes and reacted with Vulcan Fast Red (BioCare Medical, CA) substrate. All sections are counterstained with Gill's hematoxylin.

1. Leroy, E. M. et al. Fruit bats as reservoirs of Ebola virus. Nature 438, 575-576 (2005).

2. Barette, R. W. et al. Discovery of swine as a host for the Reston ebolavirus. Science 325, 204-206 (2009).

3. WHO, Ebola Reston in pigs and humans, Philippines. Weekly Epidemiological Record 7, 47-50 (2009).

4. Marsh, G. A. et al. Ebola Reston virus infection in pigs: clinical significance and transmission potential. J. Infect. Dis. 204 (Suppl.3), S804-S809 (2011).

5. Jahrling, P. B. et al. Experimental infection of cynomolgus macaques with EbolaReston filoviruses from the 1989-1990 U.S. epizootic. Arch Virol Suppl 11, 115-134 (1996).

6. Kobinger, G. P. et al. Replication, pathogenicity, shedding, and transmission of Zaire ebolavirus in pigs. J. Infect. Dis. 204, 200-208 (2011).

7. Feldmann, H. \& Geisbert, T. W. Ebola haemorrhagic fever. The Lancet 377, 849-862 (2011)

8. Dalgard, D. W. et al. Combined simian hemorrhagic fever and Ebola virus infection in cynomolgus monkeys. Lab Anim. Sci. 42, 152-157 (1992).

9. Jaax, N. et al. Transmission of Ebola virus (Zaire strain) to uninfected control monkeys in a biocontainment laboratory. The Lancet 346, 1669-1671 (1995).

10. Johnson, E., Jaax, N., White, J. \& Jahrling, P. Lethal experimental infections of rhesus monkeys by aerosolized Ebola virus. Int. J. Exp. Path. 76, 227-236 (1995)

11. Roels, T. H. et al. Ebola hemorrhagic fever, Kikwit, Democratic Republic of the Congo (1995: Risk factors for patients without a reported exposure. J. Infect. Dis. 179 (Suppl.1), S92- S97 (1999).

12. Baskerville, A., Bowen, E. T., Platt, G. S., McArdell, L. B. \& Simpson, D. I. The pathology of experimental Ebola virus infection in monkeys. J. Pathol. 125, 131-138 (1978).

13. Jaax, N. K. et al. Lethal experimental infection of rhesus monkeys with Ebola-Zaire (Mayinga) virus by the oral and conjunctival route of exposure. Arch. Pathol. Lab. Med. 120, 140-55 (1996).

14. Larsen, T. et al. Pathologic findings associated with delayed death in nonhuman primates experimentally infected with Zaire Ebola virus. J. Infect. Dis. 196 Suppl 2, S323-S328 (2007).

15. Geisbert, T. W. et al. Vesicular stomatitis virus-based vaccines protect nonhuman primates against aerosol challenge with Ebola and Marburg viruses. Vaccine 26, 6894-6900 (2008).

16. Geisbert, T. W. et al. Postexposure protection of non-human primates against a lethal Ebola virus challenge with RNA interference: a proof-of-concept study. Lancet 375, 1896-905 (2010).

17. Donaldson, A. I. \& Alexandersen S. Predicting the spread of foot and mouth disease by airborne virus. Rev Sci Tech OIE 21, 569-575 (2002).

\section{Acknowledgement}

We would like to thank Dr. Melanie van der Loop, Kevin Tierney, and Gary Wong for the assistance with animal care, to Peter Marszal, Jill Graham and Brad Collignon for the technical assistance, and to Dr. Soren Alexandersen for the critical review of the manuscript. The project was supported by CFIA and PHAC, with funding provided from the CRTI Cluster Activity fund CRTI-3780-2011-30va-17.

\section{Author contributions}

H.M.W. and G.K. conceived the study, design experiments, performed the animal experiments, analyzed and interpreted data, and wrote the manuscript. C.E-H. provided analysis of histopathology and data interpretation; A.L., G.S. and C.N. performed in vitro experiments and analyzed related data.

\section{Additional information}

Supplementary information accompanies this paper at http://www.nature.com/ scientificreports

Competing financial interests: The authors declare no competing financial interests.

License: This work is licensed under a Creative Commons

Attribution-NonCommercial-No Derivative Works 3.0 Unported License. To view a copy of this license, visit http://creativecommons.org/licenses/by-nc-nd/3.0/.

How to cite this article: Weingartl, H.M. et al. Transmission of Ebola virus from pigs to non-human primates. Sci. Rep. 2, 811; DOI:10.1038/srep00811 (2012). 